\title{
MicroRNA-153-5p promotes the proliferation and metastasis of renal cell carcinoma via direct targeting of AGO1
}

\author{
Zeyan $\mathrm{Li}^{1,2}$, Shuo Zhao ${ }^{1,2}$, Shiqin $\mathrm{Zhu}^{3}$ and Yidong Fan ${ }^{1}$
}

\begin{abstract}
MicroRNAs (miRNAs) have been demonstrated to affect the biological processes of cancers and showed great potential for prognostic biomarkers. In this study, we screened differentially expressed miRNAs in ccRCC based on three dimensions of metastasis, prognosis, and differential expression compared to normal tissue using bioinformatics algorithms. MiR-153-5p was identified as a candidate miRNA to promote $c C R C C$ occurrence and progression. Clinically, we found that miR-153-5p was significantly upregulated and related to unfavorable clinical features in ccRCC. Besides, miR-153-5p served as an independent prognostic biomarker. Functionally, miR-153-5p depletion remarkably inhibited the proliferation and metastasis of cCRCC via the phosphatidylinositol 3-kinase (PI3K)/Akt signaling. Furthermore, AGO1 was proved to be a direct target of miR-153-5p. AGO1 is associated with favorable clinical features and exhibited independent prognostic value in cCRCC. Besides, we observed that AGO1 knockdown significantly promoted tumor proliferation and metastasis. Downregulation of AGO1 partly abolished the oncogenic effects of miR-153-5p knockdown. Furthermore, miR-153-5p combined with AGO1 showed more robust prognostic significance in ccRCC. In conclusion, we found that the newly identified miR-153-5p/AGO1 axis was responsible for tumor occurrence and progression via PI3K/Akt signaling, which may therefore provide promising therapeutic targets and prognostic biomarkers for patients with cCRCC.
\end{abstract}

\section{Introduction}

Renal cell carcinoma (RCC), as the third most common urological tumor, accounts for $\sim 90 \%$ of kidney cancers and $4 \%$ of all adult malignancies ${ }^{1}$. Clear cell RCC (ccRCC) is the most common RCC subtype and rarely leads to symptoms in its early stages. However, metastatic diseases in the advanced stage instead of primary tumor impair patients' lives by decreasing the chance of 5-year survival rate from $69.4 \%$ to $10 \%$. 2 . Surgical resection is the main curative therapy for ccRCC due to its resistance to

\footnotetext{
Correspondence: Shiqin Zhu (shiqinzsq@163.com) or Yidong Fan (fanyd@sdu. edu.cn)

'Department of Urology, Qilu Hospital, Cheeloo College of Medicine,

Shandong University, Jinan, Shandong 250012, China

${ }^{2}$ Laboratory of Basic Medical Sciences, Qilu Hospital of Shandong University,

Jinan, Shandong 250012, China

Full list of author information is available at the end of the article

Edited by D. Aberdam
}

chemoradiotherapy, while $30 \%$ of patients will still experience recurrence or metastasis after nephrectomy ${ }^{4}$. As ccRCC is highly heterogeneous and intricate in molecular pathogenesis, the underlying mechanisms of initiation and metastasis remain largely elusive. Thus, exploring new clinical biomarkers and therapeutic targets is urgently required for patients with ccRCC.

MicroRNAs (miRNAs), which are small noncoding regulatory RNAs, contribute to the regulation of multiple mechanisms regarding tumorigenesis and metastasis ${ }^{5}$. miRNAs function as tumor suppressors or oncomiRNAs through downregulating the transcription of cancerrelated genes by binding directly to the target sites in its $3^{\prime}$-untranslated regions $\left(3^{\prime}-\mathrm{UTR}\right)^{6,7}$. Recent studies have reported that several miRNAs, such as miR-543, miR-223$3 \mathrm{p}$, and miR-200b, were dysregulated and showed prognostic significance in kidney tumors ${ }^{8-10}$. MiR-153-5p,

\section{(c) The Author(s) 2021}

(c) Open Access This article is licensed under a Creative Commons Attribution 4.0 International License, which permits use, sharing, adaptation, distribution and reproduction cc) in any medium or format, as long as you give appropriate credit to the original author(s) and the source, provide a link to the Creative Commons license, and indicate if changes were made. The images or other third party material in this article are included in the article's Creative Commons license, unless indicated otherwise in a credit line to the material. If material is not included in the article's Creative Commons license and your intended use is not permitted by statutory regulation or exceeds the permitted use, you will need to obtain permission directly from the copyright holder. To view a copy of this license, visit http://creativecommons.org/licenses/by/4.0/. 


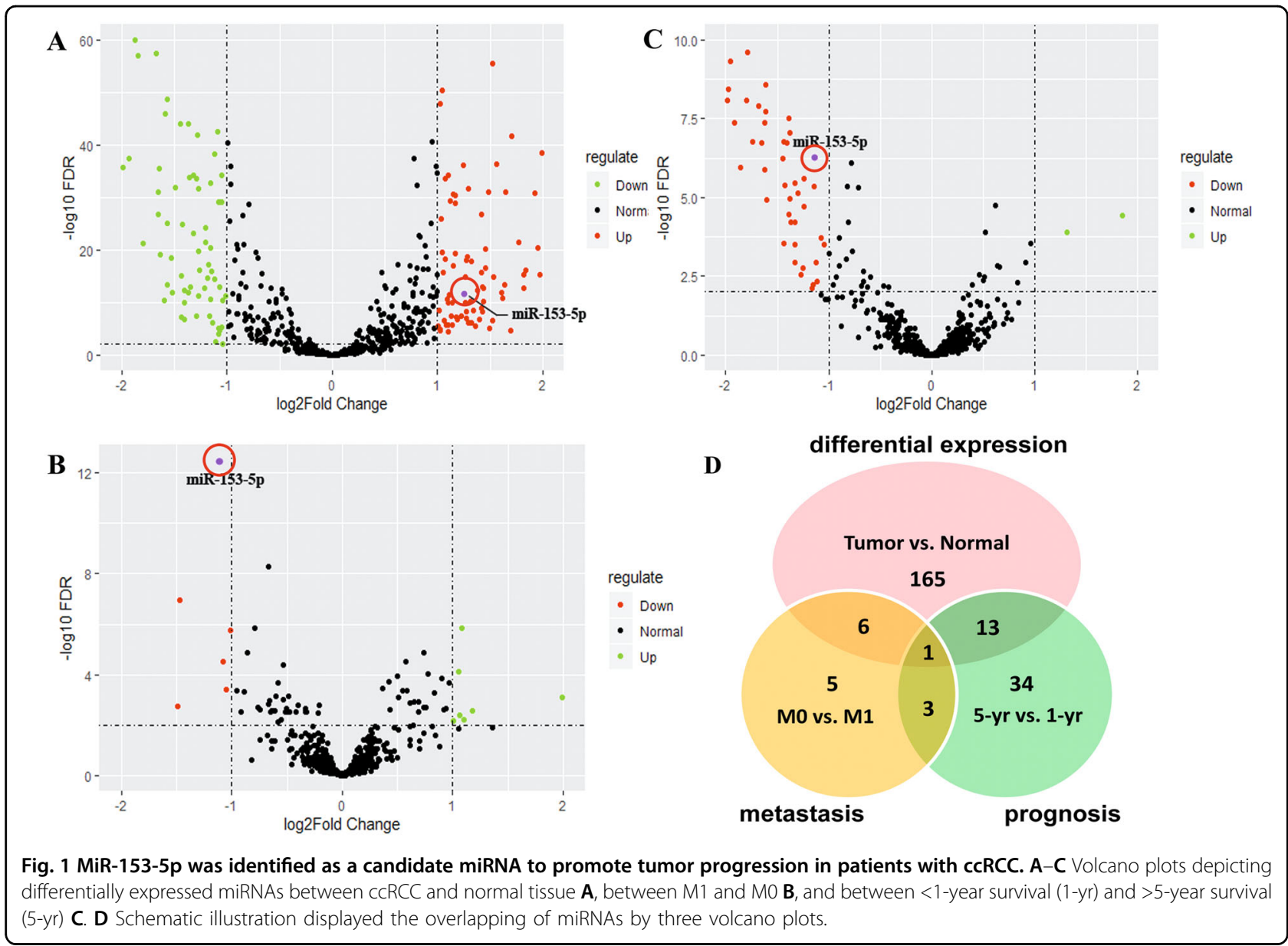

generated from the $5^{\prime}$-arm of precursor miR-153, was found to be implicated in the pathological processes of cancer. A recent study has illustrated that miR-153-5p could abolish circPAN3-induced promotion of chemoresistance in acute myeloid leukemia ${ }^{11}$. Interestingly, as the $3^{\prime}$-arm of precursor miR-153, miR-153-3p was reported to be downregulated and function as a tumor suppressor via targeting ZEB2 and Uc.416 + A in $\mathrm{ccRCC}^{12,13}$. However, given the complicated function and regulation of miRNA, the definite biological role of miR153-5p underlying ccRCC remains to be fully investigated.

In our study, we systematically screened metastasisrelated, prognosis-related, and differentially expressed miRNA in ccRCC using bioinformatics algorithms. MiR153-5p was identified as overexpressed in ccRCC and closely related to poor prognosis and metastasis. We first demonstrated the oncogenic role of miR-153-5p and its underlying molecular mechanism in ccRCC. Moreover, we initially verified argonaute (AGO) RNA-induced silencing complex (RISC) catalytic component 1 (AGO1) as the direct target of miR-153-5p and the function of AGO1 as a tumor suppressor in ccRCC. According to our findings, we proposed that miR-153-5p combined with AGO1 may be a promising clinical prognostic assessment tool and therapeutic target.

\section{Results}

MiR-153-5p was identified as a candidate miRNA to promote tumor progression in patients with $\mathrm{CCRCC}$

To explore the ccRCC progression-related miRNA, we performed the differential expression analyses of miRNA using The Cancer Genome Atlas (TCGA) database through bioinformatics algorithms. The analyses were based on the three dimensions of metastasis, prognosis, and differential expression (vs. normal tissue) in ccRCC. For the differential analyses of metastasis, we divided the ccRCC patients into metastasis (M1) group and nonmetastasis (M0) group, whereas for that of prognosis, the patients were allocated to $<1$-year survival group and $>5$ year survival (5-yr) group. Volcano plots showed that 185 miRNAs differentially expressed for differential expression groups (Fig. 1A), 15 miRNAs for metastasis groups (Fig. 1B), and 51 miRNAs for prognosis groups (Fig. 1C) with the filter of 2-folds. Among all the differentially expressed miRNAs, miR-153-5p occurred simultaneously in three volcano plots (Fig. 1D). Results indicated that 


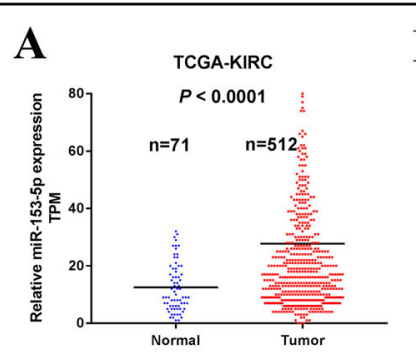

E

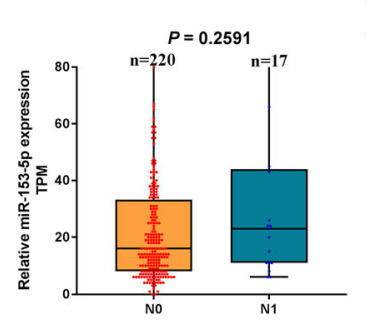

\section{B}

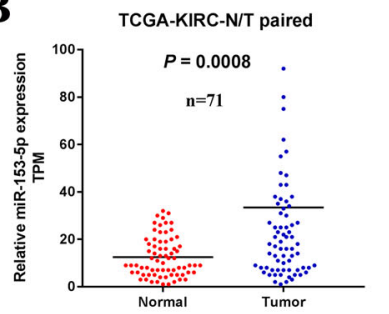

F

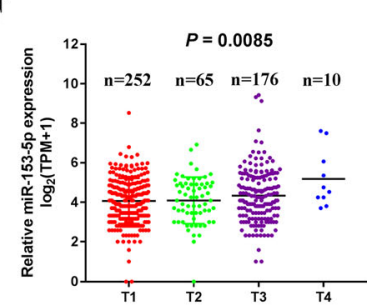

C

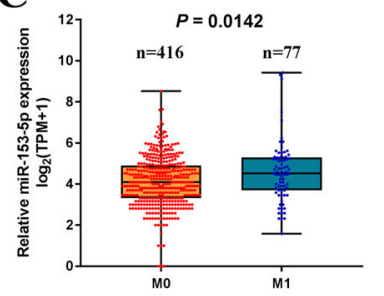

G

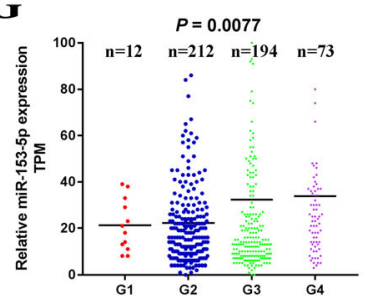

D

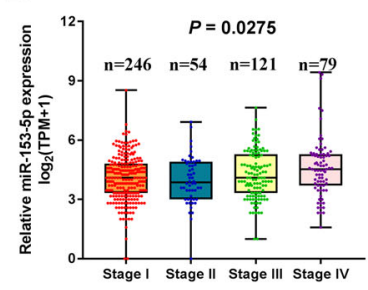

H

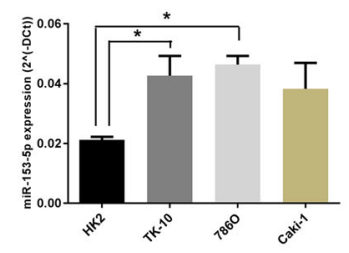

I

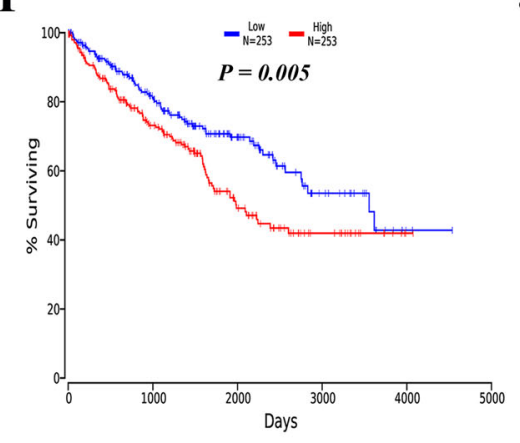

J

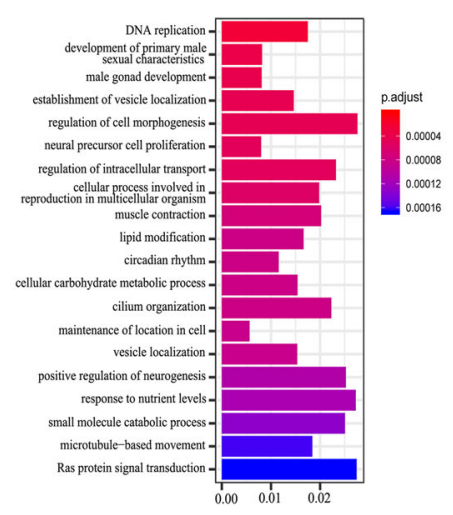

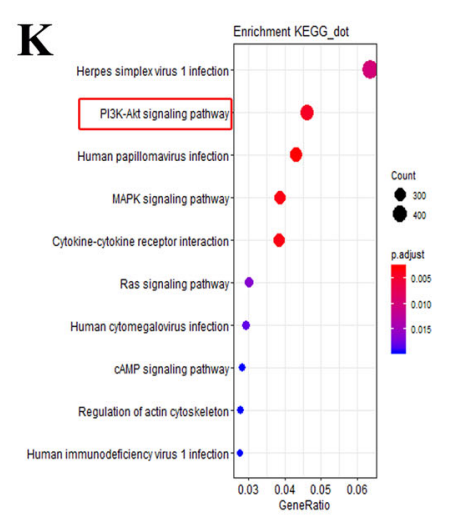

Fig. 2 MiR-153-5p is aberrantly overexpressed in ccRCC and related to unfavorable clinical features and prognosis. A, B Relative expression of miR-153-5p in tumor-normal tissues (A) and paired tumor-normal tissues (B) of TCGA. Horizontal lines represent the mean value. C-G Correlation of miR-153-5p with clinicopathological characteristics, including metastasis $(\mathbf{C})$, TNM stage (D), lymph node metastasis $(\mathbf{E})$, T stage $(\mathbf{F})$, and histologic grade $(\mathbf{G})$. $\mathbf{H}$ The expression level of miR-153-5p in ccRCC cell lines and human renal tubular epithelial cells. Horizontal lines in $\mathbf{F}$, G represent the mean value. Horizontal lines in the boxes in $\mathbf{C}-\mathbf{E}$ indicated the median value. Boxes span the interquartile range. Whiskers extend from the minimum to the maximum. The values in $\mathbf{H}$ are presented as mean \pm SEM. I Kaplan-Meier analysis of the correlation between miR-153-5p and overall survival in ccRCC. J, K Gene Ontology (GO) enrichment analysis (J) and Kyoto Encyclopedia of Genes Genomes (KEGG) pathways enrichment analysis (K) for the expression of miR-153-5p. All experiments were conducted at least three independent times. ${ }^{*} P<0.05$.

miR-153-5p was significantly overexpressed in ccRCC tissue and downregulated in M0 groups as well as in the 5-yr group. Thus, we identified miR-153-5p as a candidate miRNA promoting ccRCC progression.

MiR-153-5p is related to unfavorable clinical features and serves as an independent prognostic biomarker in cCRCC

We analyzed the correlation of miR-153-5p with clinicopathological characteristics and overall survival in ccRCC. As shown in Fig. 2, the expression levels of miR153-5p were significantly increased in ccRCC than those in normal tissue (Fig. $2 \mathrm{~A}, P<0.001$ ), and the result was consistent with that in normal-tumor pairs from the TCGA database (Fig. 2B, $P=0.0008$ ). Higher expression of miR-153-5p was observed in the M1 group compared to that in the M0 group (Fig. $2 \mathrm{C}, P=0.014$ ) and, likewise, miR-153-5p overexpression was closely related to an increased prevalence of M1 (Table 1). The upregulation of miR-153-5p was remarkably associated with the higher TNM (T: the size of tumor; N: lymph nodes involvement; M: distant metastasis) stage (Fig. 2D and Table 1). Besides, the expression levels of miR-153-5p were elevated in patients with lymph node metastasis and higher $\mathrm{T}$ stage (Fig. 2E, F). Higher miR-153-5p was found in ccRCC with advanced histological grade (Fig. $2 \mathrm{G}, P=0.008$ ) and miR153-5p overexpression showed a remarkable correlation with higher histologic grade (Table $1, P=0.001$ ). Otherwise, ccRCC cell lines increased the expression of 
Table 1 Correlations of miR-153-5p and AGO1 mRNA with clinicopathological characteristics in patients with ccRCC.

\begin{tabular}{|c|c|c|c|c|c|c|}
\hline \multirow[t]{2}{*}{ Parameter } & \multicolumn{3}{|c|}{ miR-153-5p expression } & \multicolumn{3}{|c|}{ AGO1 mRNA expression } \\
\hline & Low & High & $P$ & Low & High & $P$ \\
\hline \multicolumn{7}{|l|}{ TNM stage } \\
\hline $1+\|$ & 161 & 139 & $0.045^{*}$ & 140 & 182 & $0.001^{*}$ \\
\hline III & 62 & 59 & & 72 & 51 & \\
\hline IV & 30 & 49 & & 52 & 30 & \\
\hline \multicolumn{7}{|l|}{ T stage } \\
\hline $\mathrm{T} 1+\mathrm{T} 2$ & 166 & 151 & 0.328 & 152 & 188 & $0.001^{*}$ \\
\hline $\mathrm{T} 3+\mathrm{T} 4$ & 89 & 97 & & 113 & 77 & \\
\hline \multicolumn{7}{|l|}{ N stage } \\
\hline No & 117 & 103 & 0.340 & 114 & 125 & 0.501 \\
\hline $\mathrm{N} 1$ & 7 & 10 & & 9 & 7 & \\
\hline \multicolumn{7}{|l|}{ M stage } \\
\hline Mo & 220 & 196 & $0.025^{*}$ & 207 & 233 & $0.009^{*}$ \\
\hline M1 & 30 & 47 & & 49 & 31 & \\
\hline \multicolumn{7}{|l|}{ Grade } \\
\hline $\mathrm{G} 1+\mathrm{G} 2$ & 166 & 151 & $0.001^{*}$ & 95 & 146 & $0.0001^{*}$ \\
\hline G3 & 113 & 81 & & 108 & 98 & \\
\hline \multirow[t]{2}{*}{ G4 } & 24 & 49 & & 55 & 20 & \\
\hline & ${ }^{\mathrm{a}}$ Cox coefficient & FDR corrected ${ }^{a}$ & $P^{a}$ & & & \\
\hline miR-153-5p & 0.245 & 0.0406 & $0.0039^{*}$ & & & \\
\hline AGO1 & -0.231 & 0.021 & $0.006^{*}$ & & & \\
\hline
\end{tabular}

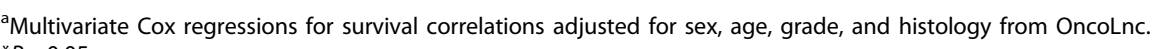

${ }^{*} P<0.05$.

miR-153-5p compared to human renal tubular epithelial cells (Fig. 2H).

Next, to investigate the prognostic significance of miR153-5p, Kaplan-Meier survival analysis was employed according to the dataset from OncoLnc ${ }^{14}$. Results showed that low expression of miR-153-5p contributed to a favorable overall survival in ccRCC (Fig. $2 \mathrm{I}, n=506, P=$ 0.005). Moreover, multivariate Cox regressions demonstrated that the prognostic impact of miR-153-5p remained significant after adjustment for sex, age, grade, and histology (Table 1, Cox Coefficient $=0.245, P=$ 0.004). These findings indicated that miR-153-5p was an independent prognostic biomarker in ccRCC. What's more, the Kyoto Encyclopedia of Genes Genomes (KEGG) pathways enrichment analysis and Gene Ontology (GO) analysis were performed to study the miR-153-5p-related signaling. Results showed that miR-153-5p was mainly enriched in DNA replication, proliferation, and other functions, and closely associated with PI3K/Akt signaling pathway (Fig. 2J, K).
MiR-153-5p promotes the proliferation and metastasis via the PI3K/Akt pathway

To explore the role of miR-153-5p on the proliferation and metastasis of ccRCC, we transfected 786-O and TK10 with miR-153-5p inhibitor or NC inhibitor, and performed the Cell counting kit 8 (CCK-8) assay, transwell assay, and wound-healing assay, to examine the effects of miR-153-5p. Results showed that the expression level of miR-153-5p was remarkably decreased after miR-153-5p depletion (Fig. 3A). MiR-153-5p knockdown significantly downregulated cell proliferation rate (Fig. 3B). Transwell assay showed that the migration and invasion ability of ccRCC cells obviously decreased after transfection with miR-153-5p inhibitor (Fig. 3C, D). Likewise, miR-153-5p upregulation with miR-153-5p mimics significantly elevated proliferation, migration, and invasion of ccRCC (Fig. S1). Besides, cell proliferation did not significantly change after transfection with miR-153-5p inhibitor or mimics under serum-free culture conditions (Fig. S2). Thus, the impact of proliferation on invasion and migration could 
A

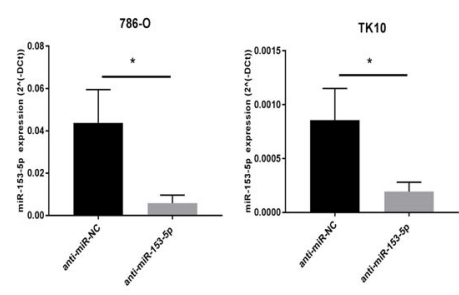

C

Migration

786-0

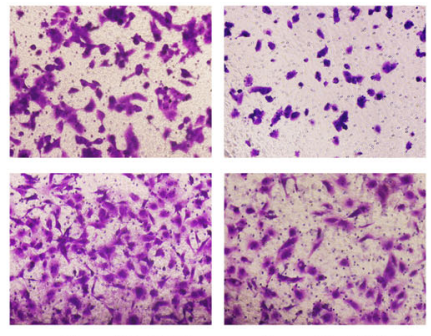

anti-miR-NC
B

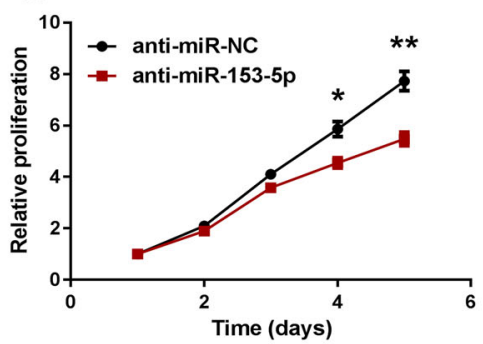

Invasion

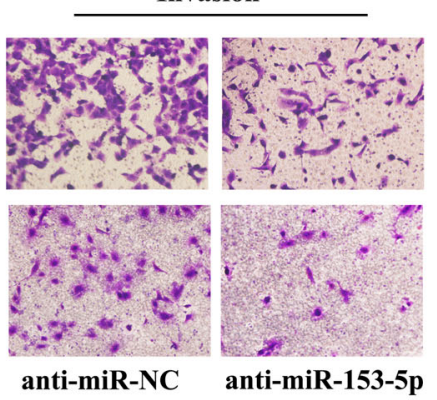

TK10
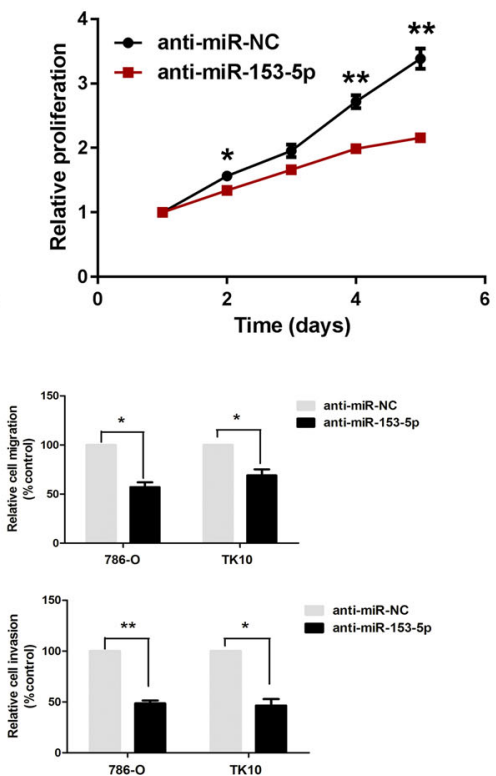

KK10
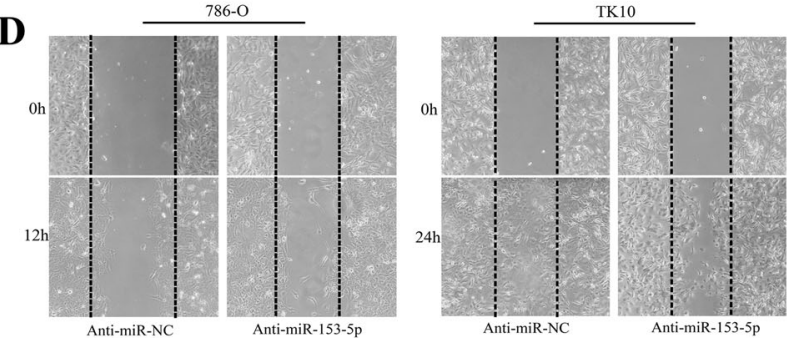

$\mathbf{E}$

- 786-0

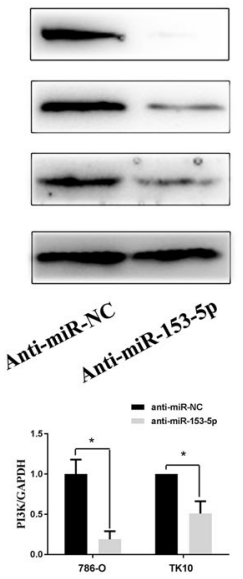

TK10

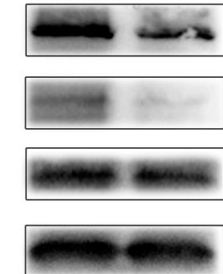

PI3K (110 KDa)

p-Akt (60 KDa)

Akt (60 KDa)

GAPDH (37 KDa)
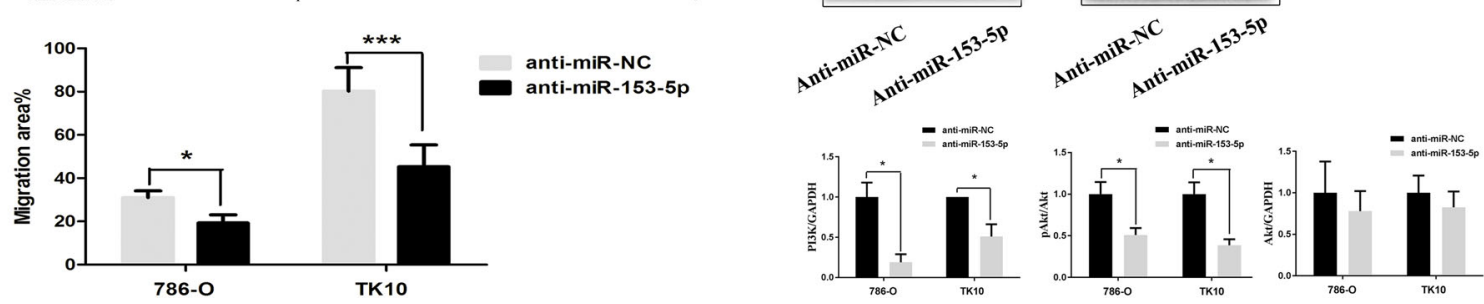

Fig. 3 MiR-153-5p knockdown decreased proliferation and metastasis of ccRCC via PI3K/Akt signaling. A The expression level of miR-153-5p in 786-O and TK10 after transfection with miR-153-5p inhibitor. B The proliferation rate of ccRCC after the miR-153-5p knockdown. C, D Effect of miR153-5p depletion on migration (C, D) and invasion (C). E Effect of miR-153-5p depletion on PI3K/Akt signaling. All experiments were conducted at least three independent times and all the values were presented as mean \pm SEM. ${ }^{*} P<0.05,{ }^{* *} P<0.01,{ }^{* * *} P<0.001$.

be ruled out. Further investigation of the underlying mechanism of miR-153-5p, we found that miR-153-5p knockdown could significantly reduce the relative expression level of PI3K and p-Akt. Meanwhile, miR-153$5 p$ depletion showed no effect on the relative expression level of Akt. These results indicated that the PI3K/Akt pathway was involved in the stimulative effects of miR153-5p on proliferation and metastasis (Fig. 3E).

\section{AGO1 is a direct target of miR-153-5p}

To investigate the underlying molecular mechanism that was involved in the oncogenic role of miR-153-5p, we screened the potential gene targets of miR-153-5p based on the bioinformatics algorithms of TargetScan, miRWalk, microT-CDS, miRPathDB, and miRDB. A total of 77 genes was overlapped with high stringency (Fig. 4A). We further identified the candidate target genes according to the following criteria: (1) prognosis-related and metastasis-related genes in ccRCC based on GEPIA and LinkedOmics; and (2) had the definite function of tumor suppressor based on literature review. Finally, the top six genes meeting all these above requirements were tested, including AGO1, coiled-coil domain containing 68 (CCDC68), cell division cycle 73 (CDC73), phosphatase 


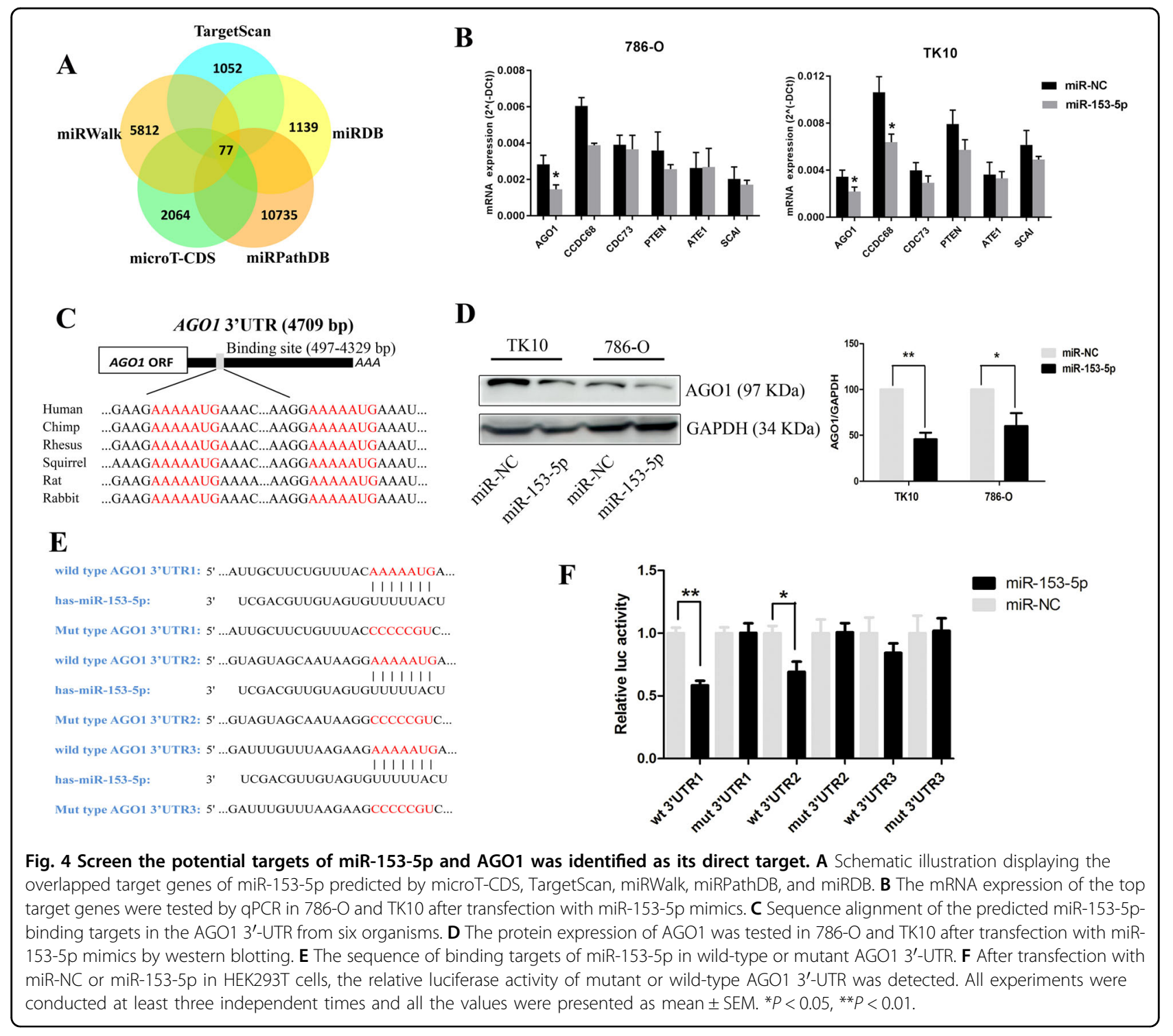

and tensin homolog deleted on chromosome ten (PTEN), arginyltransferase 1 (ATE1), and suppressor of cancer cell invasion (SCAI). Next, quantitative PCR (qPCR) was performed to test their mRNA expression. We found AGO1 and CCDC68 were significantly downregulated in 786-O after transfection with miR-153-5p, whereas AGO1 were downregulated in TK10 (Fig. 4B). AGO1 was overlapped in two cell lines and CCDC68 was only significantly downregulated in TK10 cells. However, CCDC68 knockdown did not show an obvious effect on proliferation in ccRCC (Fig. S3). Thus, we speculated that AGO1 may be the potential target of miR-153-5p.

As shown in Fig. 4C, the sequence of the putative bindings are in 497-504, 1623-1630, and 4322-4329 bp of AGO1 3'-UTR. The Homology search demonstrated that putative targets are evolutionarily conserved. The protein expression of AGO1 was remarkably decreased after overexpression of miR-153-5p (Fig. 4D). Subsequently, to explore whether miR-153-5p could directly bind to AGO1 $3^{\prime}$-UTR, we cloned the sequence of AGO1 $3^{\prime}$-UTR flanked three wild type or three mutant-binding targets of miR-153-5p into the pmiRGLO vector, individually (Fig. 4E). We co-transfected HEK293T cells with miR-NC or miR-153-5p and pmiRGLO-3'-UTR vectors. As illustrated in Fig. 4F, the relative luciferase activities of wild-type AGO1 $3^{\prime}$-UTR1 and 3'-UTR2 vectors were significantly reduced after transfection with miR-153-5p. On the contrary, the decrease of luciferase activity was fully abolished when mutant AGO1 $3^{\prime}$-UTR1 and $3^{\prime}$ UTR2 were co-transfected with miR-153-5p. However, the luciferase activity of wild-type AGO1 3'-UTR3 did not significantly downregulated when co-transfected with miR-153-5p. Collectively, these results demonstrated that AGO1 serves as a direct target of miR-153-5p. 

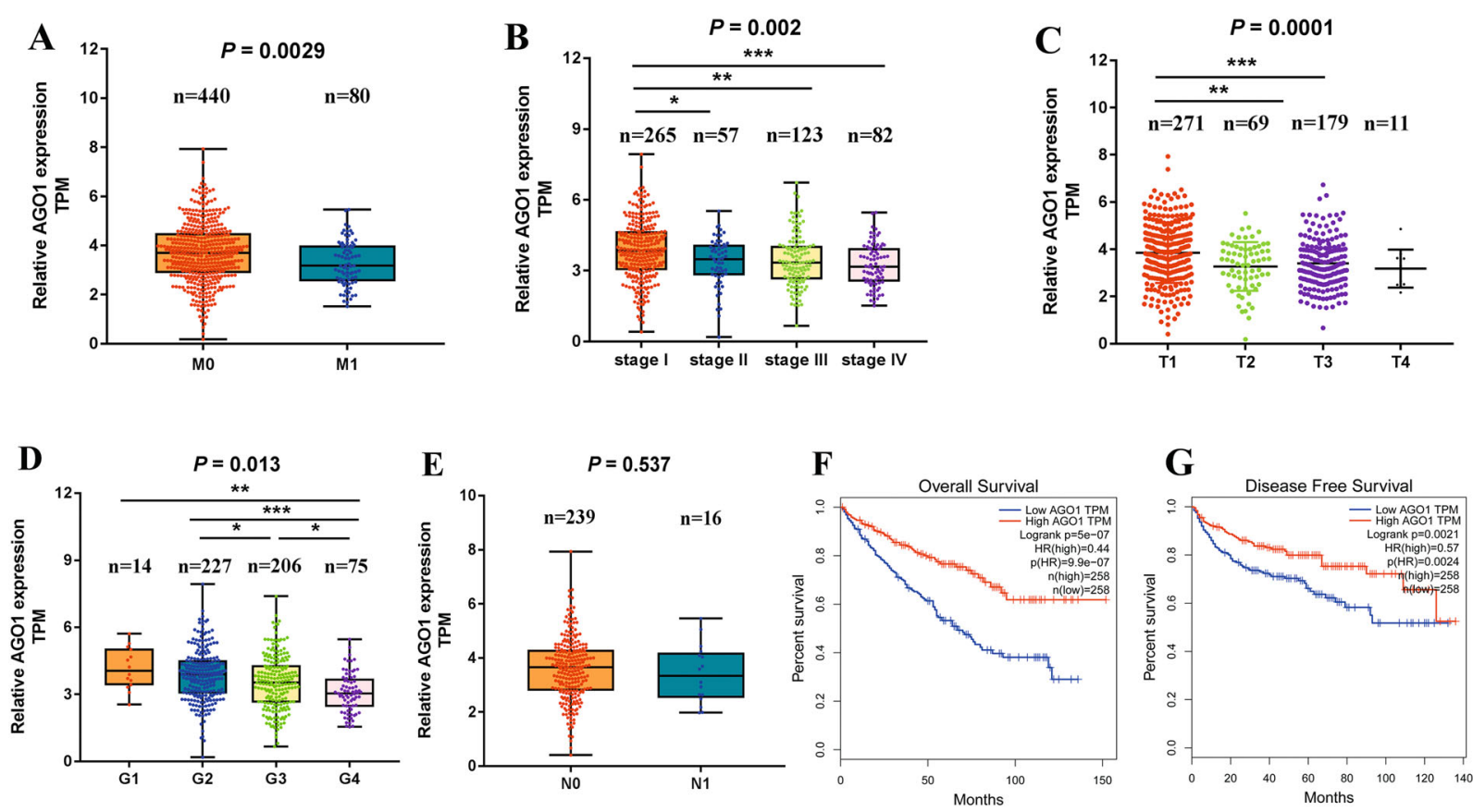

Fig. 5 AGO1 is closely related to favorable clinical features and prognosis. A-E Correlation of AGO1 with clinicopathological characteristics in patients with ccRCC, including metastasis (A), TNM stage (B), T stage $(\mathbf{C})$, histologic grade (D), and lymph node metastasis $(\mathbf{E})$. Horizontal lines in the boxes in $\mathbf{A}, \mathbf{B}, \mathbf{D}, \mathbf{E}$ indicated the median value. Boxes spanned the interquartile range. Whiskers extended from the minimum to the maximum. Horizontal lines in $\mathbf{C}$ represent the mean value. $\mathbf{F}$, G Kaplan-Meier analysis of overall survival $(\mathbf{F})$ and disease-free survival $(\mathbf{G})$ regarding AGO1 expression in $\mathrm{CCRCC}$ patients. ${ }^{*} P<0.05,{ }^{* *} P<0.01,{ }^{* *} P<0.001$.

AGO1 is associated with favorable clinical features and serves as an independent prognostic biomarker in cCRCC

To demonstrate the clinical significance of AGO1 in ccRCC, we investigated the correlation of AGO1 expression with clinicopathological features based on the TCGA dataset. The results showed that the expression levels of AGO1 were significantly decreased in patients with M1 than that in patients with M0 (Fig. 5A, $P=0.003$ ) and similarly AGO1 overexpression was related to a decreased prevalence of M1 (Table 1, $P=0.009$ ). Besides, lower expression of AGO1 was found in higher TNM stage (Fig. $5 \mathrm{~B}, P=0.022$ ), $\mathrm{T}$ grade (Fig. $5 \mathrm{C}, P=0.0001$ ), and histological grade (Fig. 5D, $P=0.013$ ). Likewise, AGO1 overexpression had significant correlations with lower TNM stage, $\mathrm{T}$ stage, and histological grade (Table 1 , all $P<$ 0.01). AGO1 expression was decreased in patients with $\mathrm{N} 1$; however, no statistical significance was observed (Fig. $5 \mathrm{E}$ and Table 1).

As shown in Fig. 5F, G, patients with low AGO1 expression had worse overall survival (hazard ratio $(\mathrm{HR})=0.44$, $P<0.001)$ and disease-free survival $(\mathrm{HR}=0.57, P=0.002)$. Moreover, multivariate Cox regressions demonstrated that the prognostic impact of AGO1 remained significant after adjustment for sex, age, grade, and histology (Table 1, Cox coefficient $=-0.231, \quad P=0.006)$, suggesting that
AGO1 serves as an independent prognostic biomarker in ccRCC.

The role of miR-153-5p on tumor progression is mediated via downregulation of AGO1

Our results have demonstrated that AGO1 was the direct target of miR-153-5p; we further determined whether AGO1 serves as a functional target gene. Small iterfering RNA (siRNA) of AGO1 (si-AGO1) was employed and the expression of AGO1 was obviously decreased after si-AGO1 treatment (Fig. 6A and Fig. S4). As is shown in Fig. 6B, C, AGO1 knockdown significantly elevated the proliferation, migration, and invasion of ccRCC. Besides, transfection with miR-153-5p inhibitor and/or si-AGO1 showed no obvious effects on the cell proliferation under serum-free culture conditions (Fig. S2). Thus, the impact of proliferation on invasion and migration could be ruled out. Next, we investigated whether the role of miR-153-5p on tumor progression was mediated via downregulation of AGO1. 786-O and TK10 cells were co-transfected with miR-153-5p inhibitor or NC inhibitor and si-AGO1 or NC. Results showed that proliferation, migration, and invasion were remarkably downregulated by the effect of miR-153-5p knockdown, which was significantly reversed by co-transfection with 
A

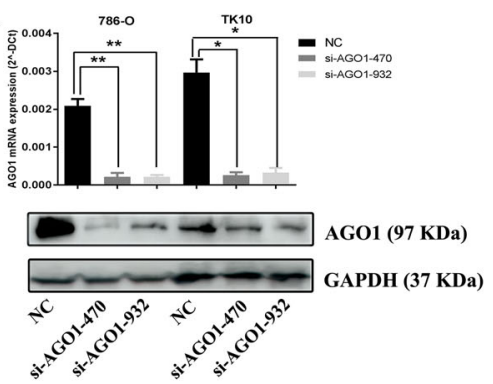

C
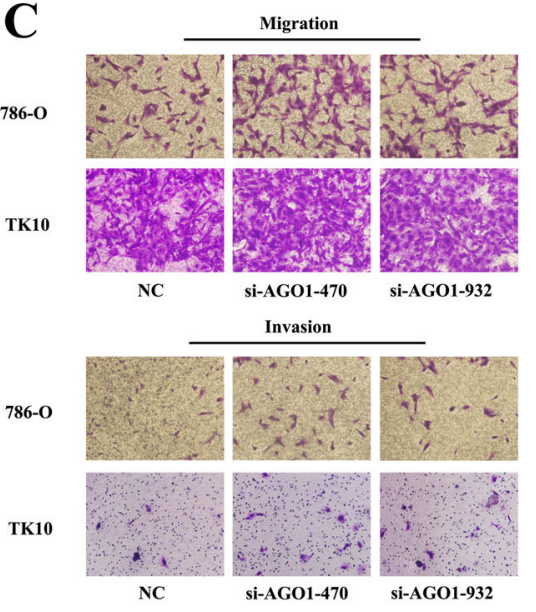

$\mathbf{E}$
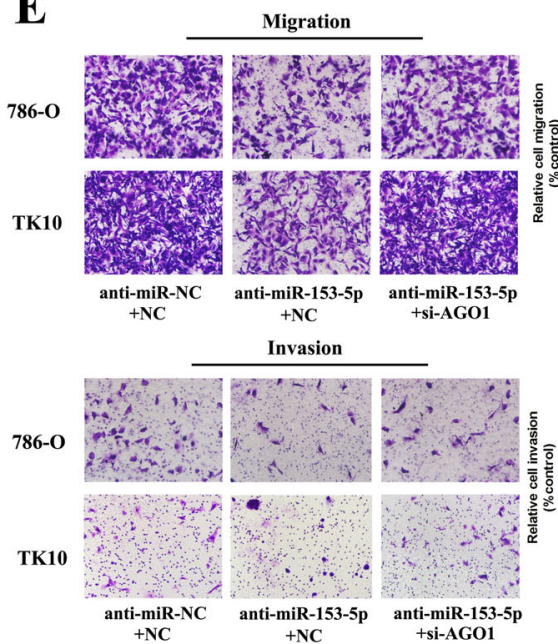
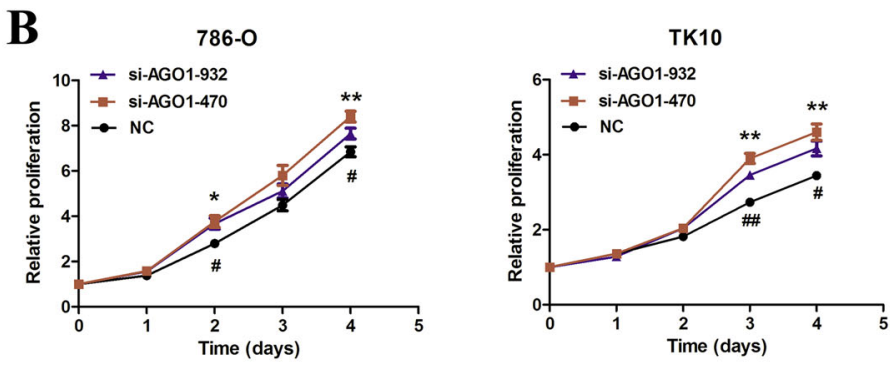

D
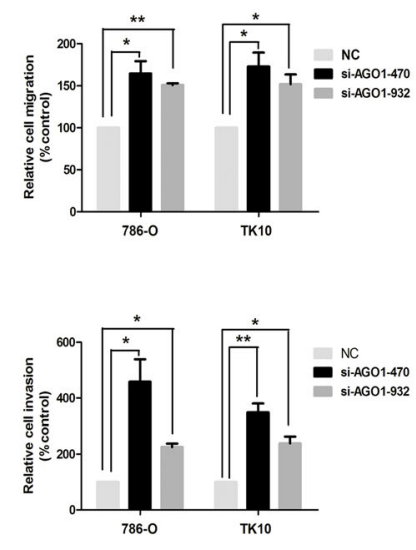

786-0

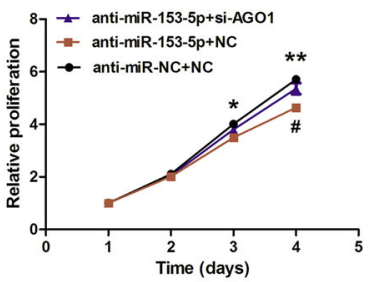

TK10

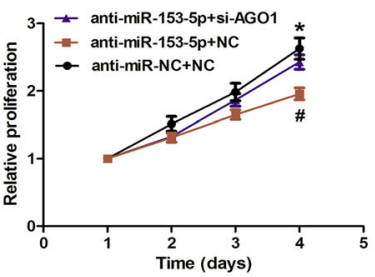

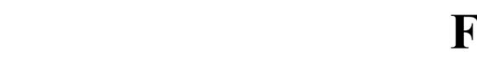

F

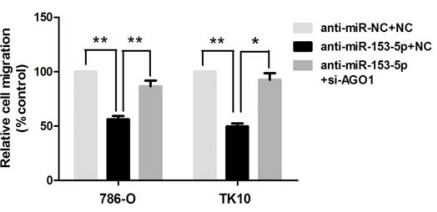

786-O TK10
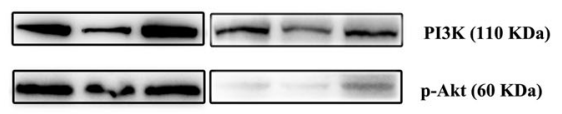

$\square-\infty-\infty$ Akt (60 KDa)

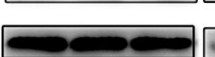

anti-miR-153-5p - + + +
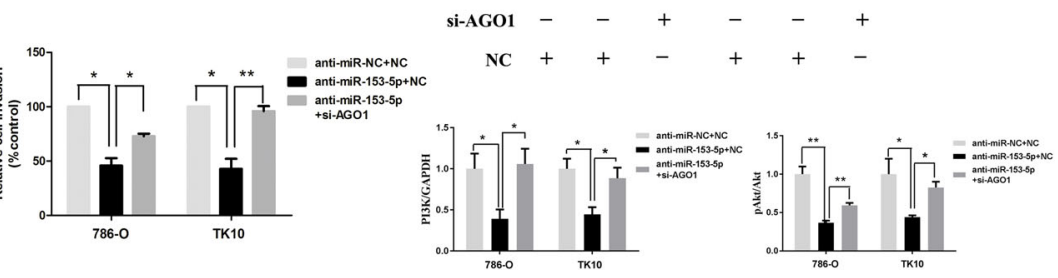

Fig. 6 The role of miR-153-5p on tumor progression is mediated via the downregulation of AGO1. A The expression level of mRNA and protein of AGO1 in 786-O and TK10 after transfection with siRNA of AGO1. B The proliferation rate of ccRCC after AGO1 knockdown. C Effect of AGO1 knockdown on migration and invasion. The proliferation (D) and metastasis (E) of ccRCC after co-transfection with miR-153-5p inhibitor or NC inhibitor with either AGO1 siRNA or NC. F The alterations of the PI3K Akt pathway after co-transfection with miR-153-5p inhibitor or NC inhibitor with either AGO1 siRNA or NC. All experiments were conducted at least three independent times and all the values were presented as mean \pm SEM. ${ }^{*} P<0.05,{ }^{* *} P<0.01$ (B vs. NC; D vs. anti-miR-153 + NC); ${ }^{\#} P<0.05,{ }^{\# \# P<0.01 ; ~ B ~ v s . ~ s i-A G 01-932 ; ~ D ~ v s . ~ a n t i-m i R-153-5 p ~+~ s i-A G O 1) . ~}$

si-AGO1 (Fig. 6D, E). To further explore the underlying mechanism of the AGO1-mediated effect of miR-153-5p, PI3K/Akt pathway was examined when cells were cotransfected with miR-153-5p inhibitor and si-AGO1. Results showed that the PI3K/Akt pathway was obviously suppressed by the effect of miR-153-5p knockdown, which was significantly reversed by co-transfection with si-AGO1 (Fig. 6F and Fig. S4). Taken together, we concluded that AGO1 is a direct functional target of miR153-5p. 


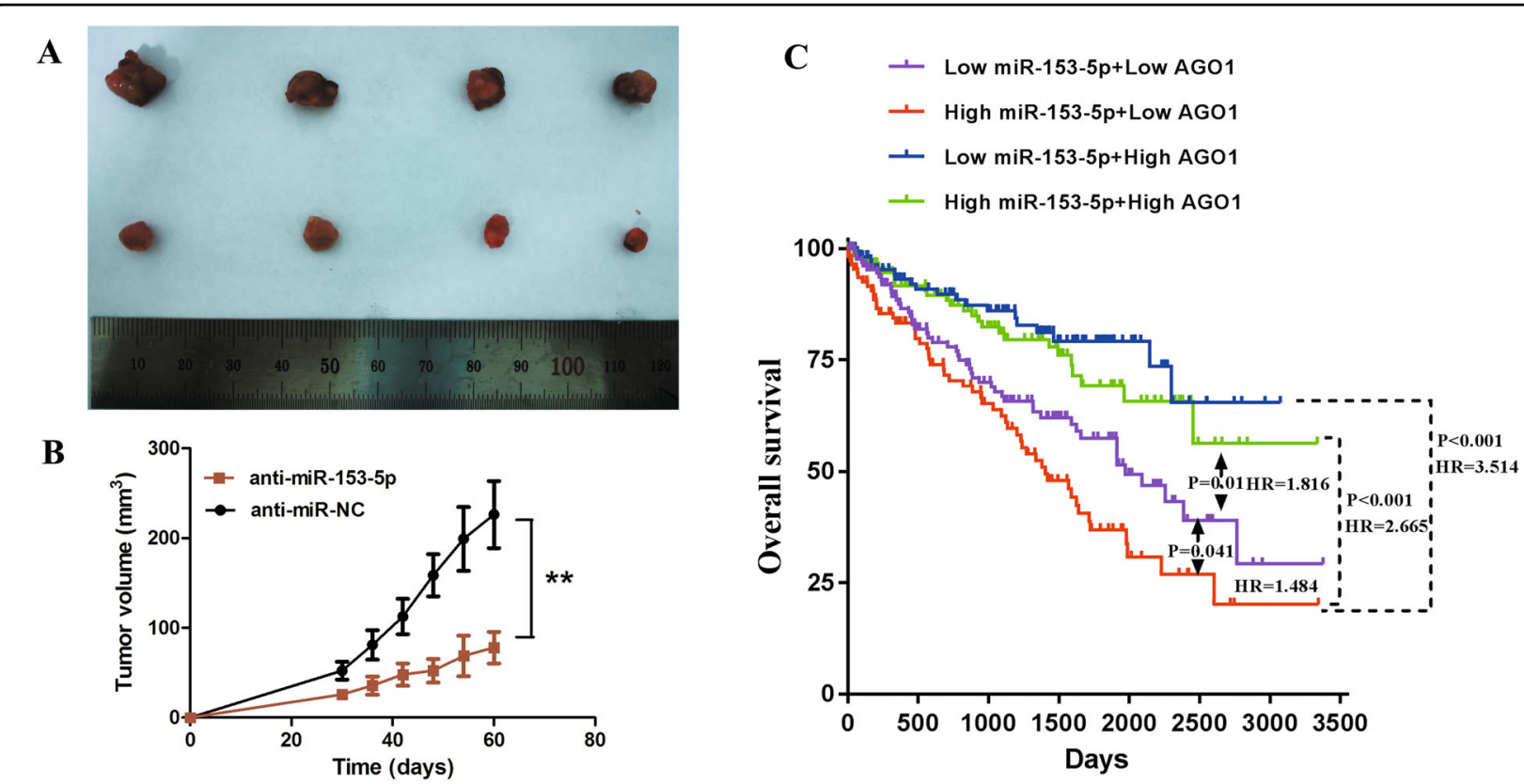

Fig. 7 MiR-153-5p knockdown inhibited tumorigenesis in vivo and miR-153-5p/AGO1 axis serves as a robust prognostic indicator in cCRCC. A, B The tumor growth of 786-O cells infected with LV3-hsa-miR-153-5p inhibitor sponge or LV3-NC. C Prognosis analysis stratified by the expression level of miR-153-5p and AGO1 according to the TCGA dataset. All experiments were conducted at least three independent times and all the values were presented as mean \pm SEM. ${ }^{* *} P<0.01$.

MiR-153-5p depletion inhibited tumorigenesis in vivo and the miR-153-5p/AG01 axis serves as a robust prognostic indicator in $\mathrm{CCRCC}$

We performed in vivo tumor growth assay to validate the effect of miR-153-5p in vivo. 786-O cells infected with LV3-hsa-miR-153-5p inhibitor sponge or LV3-NC were injected subcutaneously into the right armpit of mice. Results showed that the growth speed of tumors in the anti-miR-NC group was significantly faster than that in the anti-miR-153-5p group (Fig. 7A, B). Besides, we conducted a prognosis analysis stratified by the expression level of miR-153-5p and AGO1 according to the TCGA dataset (Fig. 7C). We found that high miR-153-5p + low AGO1 group had the worst prognosis (vs. low miR-153$5 \mathrm{p}+$ high AGO1 group: $P<0.001, \mathrm{HR}=3.51$; vs. high miR-153-5p + high AGO1 group: $P<0.001, H R=2.67$; vs. low miR-153-5p + low AGO1 group: $P=0.04, \mathrm{HR}=$ 1.48), indicating miR-153-5p/AGO1 axis serves as a robust prognostic indicator in ccRCC.

\section{Discussion}

The clinical intervention of advanced ccRCC remains a challenge because the metastatic diseases commonly become resistant to radical nephrectomy ${ }^{15}$. Due to the resistance to chemoradiotherapy of ccRCC, exploring novel molecular targets are urgently needed ${ }^{16,17}$. MiRNAs have been demonstrated to take part in the regulation of the occurrence and progression of cancers ${ }^{18,19}$ and showed great potential for the prognostic biomarkers ${ }^{20}$. In the present study, we systematically screened differentially expressed miRNAs based on three dimensions of metastasis, prognosis, and differential expression (vs. normal tissue) in ccRCC using bioinformatics algorithms. MiR153-5p was identified as a candidate oncomiRNA and showed independent prognostic significance in ccRCC. We found miR-153-5p knockdown remarkably inhibited ccRCC cell invasion, migration, and proliferation. Suppression of PI3K/Akt signaling was observed after knockdown of miR-153-5p. Moreover, AGO1 was verified to be a direct target of miR-153-5p and exhibited independent prognostic value. Downregulation of AGO1 significantly promoted the proliferation and metastasis of ccRCC. In the rescue experiments, AGO1 knockdown could partly abolish the inhibition of proliferation and metastasis and reverse the suppression of PI3K/Akt signaling induced by knockdown of miR-1535p. Furthermore, miR-153-5p combined with AGO1 may be a promising clinical prognostic assessment tool.

Mir-153-2, located on chromosome 7q36.3, was recently demonstrated to be implicated in the pathogenesis of numerous solid tumors or hematological malignancies ${ }^{11,21-24}$. However, the majority of these researches focused on the function of miR-153-3p, the $3^{\prime}$-arm of precursor miR-153, on various cancers including 
$\mathrm{RCC}^{12,13}$. MiR-153-5p, as the homological fragment of miR-153-3p, its related studies are rare and the role of miR-153-5p on ccRCC remains to be explored. Our results showed that miR-153-5p was significantly upregulated in the ccRCC tissues and cell lines. MiR-153-5p depletion remarkably inhibited ccRCC cell proliferation and metastasis. Moreover, miR-153-5p is related to unfavorable clinical features and serves as an independent prognostic biomarker in ccRCC. These findings suggested that miR-153-5p played a crucial role in tumorigenesis and progression.

To further investigate the signaling pathway involved in the oncogenic effects of miR-153-5p, we performed GO and KEGG analysis based on the TCGA dataset. MiR153-5p was mainly enriched in PI3K/Akt signaling. PI3K/ Akt signaling, which plays a pivotal role in the regulation of cell proliferation and metabolism, serves as a crucial growth regulatory pathway in tumors. PI3K/AKT pathway could promote G1/S transition by upregulation of C-myc and Cyclin $\mathrm{D}^{25}$. Apoptosis and autophagy of tumor cells were induced via deactivation of PI3K/AKT ${ }^{26,27}$. Moreover, a previous study has shown that PI3K/AKT was implicated in EMT-related metastasis by overexpression of Slug and Snail meanwhile downregulation of E-cadherin ${ }^{26}$. Clinically, PI3K/AKT signaling is highly activated in ccRCC and several of its targeted drugs have been approved for patients with $\mathrm{ccRCC}^{28}$. In the present study, we found that the PI3K/AKT pathway was significantly inhibited after miR-153-5p depletion, indicating that PI3K/AKT signaling may be involved in the oncogenic effect of miR-153-5p.

AGO1, also known as eukaryotic initiation factor $2 \mathrm{C} 1$, is one of the members of the AGO protein family (AGO1-AGO4) $^{29}$. AGOs are the core proteins of the miRNA-induced silencing complex ${ }^{30}$ and also exist in all the RISCs ${ }^{31}$, which leads to mRNA degradation and translation inhibition ${ }^{30,32}$. Among the AGO protein family, AGO1 is not only implicated in small RNA-induced gene silencing but also responsible for the unwinding of the miRNA duplexes ${ }^{33,34}$. Several investigations have reported that AGO1 played a role in various cancers. AGO1 was found to suppress tumor angiogenesis and predict a better prognosis via translational inhibition of vascular endothelial growth factor (VEGF) ${ }^{35}$. It has been reported AGO1 acted as a biomarker for colon cancer, melanoma, and muscle-invasive bladder carcinomas ${ }^{36-38}$. However, the role of AGO1 in ccRCC is still unknown. Here we first found AGO1 was a direct target of oncomiRNA miR-153$5 \mathrm{p}$ and served as an independent biomarker in ccRCC with an HR value of 0.44 . AGO1 remarkably inhibited the proliferation and metastasis of ccRCC, and significantly abolished the oncogenic effects of miR-153-5p. Downregulation of AGO1 could reverse the inhibition of PI3K/ Akt signaling caused by miR-153-5p knockdown. These findings demonstrated that AGO1, function as a tumor suppressor gene, was responsible for the oncogenic role of miR-153-5p. Notably, survival analysis showed that ccRCC patients with high miR-153-5p and low AGO1 had the worst prognosis than other patients, indicating that miR153-5p together with AGO1 may be a promising clinical prognostic assessment tool.

The tumor microenvironment has been demonstrated to be related to metastasis, chemo-resistance, angiogenesis, and immunosuppression of cancer ${ }^{39,40}$. miRNA targeting coding gene plays an important role in the crosstalk of cancer cells with the surrounding microenvironment. Emerging evidence has indicated that several miRNAs, such as miR-221-3p and miR-126, could target VEGF signaling to mediate angiogenesis ${ }^{40,41}$. MiR770 could transform the M1 phenotype of macrophages to the M2 phenotype by regulating the polarization of macrophages and further induce immunosuppression ${ }^{39}$. Therefore, therapeutic strategies focused on miRNArelated anti-angiogenic therapy and immunotherapy may be promising. Recent studies have indicated that miR153-5p could directly target Notch $^{42}$ and regulate the angiogenesis in bladder tumors by targeting indoleamine 2,3-dioxygenase $1^{42,43}$. Thus, miR-153-5p/AGO1 may be a potential therapeutic target for the anti-angiogenic therapy and immunotherapy in ccRCC and remains to be further investigated.

Notably, ccRCC, as an immune sensitive tumor, its dissemination and metastasis may be relevant to the regulation of the immune system. Studies have demonstrated that ccRCC with bone metastasis had a worse prognosis and did not show a good respond to the immunotherapy, which suggested that immune regulation may play a role in the dissemination of ccRCC and have the potential to be a prospective novel therapeutic approach for ccRCC ${ }^{44,45}$.

In summary, we demonstrated that miR-153-5p depletion could significantly inhibit the proliferation and metastasis of ccRCC, which was mediated via PI3K/Akt signaling. AGO1 was identified as a direct functional target of miR-153-5p. Conversely, downregulation of AGO1 promoted the proliferation and metastasis of ccRCC. Moreover, miR-153-5p combined with AGO1 showed more robust prognostic significance in ccRCC. The newly identified miR-153-5p/AGO1 axis may provide promising therapeutic targets and prognostic biomarkers for patients with ccRCC.

\section{Materials and methods \\ Chemicals and reagents}

MiR-153-5p inhibitor/mimic, inhibitor/mimic NC, and AGO1 siRNA were purchased from GenePharma (Shanghai, China). The siRNAs were selected by qPCR and the siRNAs with a knockdown efficiency $<70 \%$ were 
ruled out. The sequence of siRNA was listed in Table S1. INTERFERin and jetPRIME used as transfection reagents were from Polyplus-transfection ${ }^{\circledR}$ SA (Strasbourg, France). CCK-8 was obtained from Dojindo (Kumamoto, Japan). Dulbecco's modified Eagle's medium (DMEM), RPMI 1640 Medium, McCoy's 5A modified medium, fetal bovine serum (FBS), and $0.25 \%$ Trypsin-EDTA were purchased from Gibco (Grand Island, NY, USA). Antibodies to AGO1 (\#5053), Akt (\#9272 S), p-Akt (\#9271 S), PI3K (\#13857), and GAPDH (\#5174 T) were from Cell Signaling Technology (Beverly, USA). The dual-luciferase expression vector pmirGLO and Dual-Luciferase ${ }^{\circledR}$ Reporter Assay System were obtained from Promega (Madison, WI, USA). All other reagents were from Beyotime (Shanghai, China) and Solarbio (Beijing, China), unless otherwise indicated.

\section{Cell culture}

786-O, Caki-1, HK-2, and HEK293T were obtained from American Type Culture Collection. 786-O and TK10 were cultured in RPMI 1640 medium. Caki-1 cells were maintained in McCoy's 5A modified medium. HK-2 and HEK293T were cultured in DMEM medium. Cells were cultured in the medium containing 10\% FBS, $100 \mathrm{U} /$ $\mathrm{mL}$ penicillin, and $100 \mu \mathrm{g} / \mathrm{mL}$ streptomycin, and incubated in an atmosphere of $5 \% \mathrm{CO}_{2}$ at $37^{\circ} \mathrm{C}$.

\section{CCK-8 assay}

CCK-8 assay was used to detect the cell proliferation rate. 786-O and TK10 cells were seeded in 96-well plates at a density of 4000/well. After incubation overnight, the cells were transfected with miR-153-5p inhibitor/mimic, inhibitor/mimic NC, or AGO1 siRNA. Subsequently, $10 \mu \mathrm{l} \mathrm{CCK}-8$ reagent was added into each well and incubated for $1 \mathrm{~h}$ at $37^{\circ} \mathrm{C}$ after transfection for the indicated times. The absorption value at $450 \mathrm{~nm}$ was detected on a Microplate Reader (Bio-Rad, Hercules, CA).

\section{Migration and invasion assays}

Migration and invasion assays were conducted using the transwell system (Corning Costar, Lowell, MA, USA) as previously described ${ }^{46}$. In the migration assay, $4 \times 10^{4}$ $786-\mathrm{O}$ cells or $1 \times 10^{5}$ TK10 cells were suspended in $200 \mu \mathrm{l}$ of serum-free medium and added into the upper chamber. Subsequently, $600 \mu \mathrm{l}$ complete medium was added to the lower chamber. After incubation for 24 or $36 \mathrm{~h}$, the cells that migrated through the membrane were fixed for $15 \mathrm{~min}$ with $4 \%$ paraformaldehyde and then incubated in $0.1 \%$ crystal violet for $30 \mathrm{~min}$. For the invasion assay, the procedures were exhibited in the same way as mentioned above except the membranes were coated with Matrigel (BD Bioscience, USA). The cell count was quantified in three representative fields in each independent experiment.

\section{Wound-healing assay}

The cells were seeded into the six-well plates to reach $100 \%$ confluence after transfection. Horizontal lines were drawn on the back of the plate; prior to that, a linear scratch was created on a six-well plate by $200 \mu \mathrm{l}$ pipette tips and then the detached cells were removed by phosphate-buffered saline (PBS). Subsequently, the pictures of scratch were taken under the microscope for the indicated times at magnification of $\times 200$ and the images were analyzed by ImageJ.

\section{RNA isolation and qPCR assay}

Total RNA was extracted from cells or tissues with TRIzol reagents (Invitrogen, Carlsbad, CA, USA) as the instruction of the manufacturer's protocol. For mRNA, the Prime-Script RT reagent kit (TaKaRa, Shiga, Japan) was employed to synthesize cDNA from RNA and realtime PCR was conducted with SYBR Premix Ex Taq (TaKaRa) using CFX96 Real-time PCR Detection System. miRNA was reverse transcribed by miRNA First-Strand Synthesis Kit (Sparkjade, Qingdao, China) and reversetranscription PCR was performed using miRNA Real-time PCR Kit (Sparkjade). Primers of several genes were designed including miR-153-5p, AGO1, $\beta$-actin, CCDC68, CDC73, PTEN, ATE1, and SCAI. Primer information was listed in Supplementary Table S1.

\section{Dua-luciferase reporter assay}

The 3'-UTR of AGO1 carrying different putative miR153-5p-binding sites were amplified and then cloned into pmirGLO. The miR-153-5p complementary sites with the sequence 5'-AAAAATGA-3' in AGO1 3'-UTR were singly mutated to eliminate the complementarity to miR153-5p as described previously ${ }^{47}$. Cells were seeded in 24well plates and then co-transfected with wild-type or mutated AGO1 3'-UTR constructs, and miR-153-5p mimics or NC. Forty-eight hours after transfection, the cells were harvested, and the luciferase activity was measured using the Dual-Luciferase ${ }^{\circledR}$ Reporter Assay System. Normalized luciferase activity was calculated as luciferase activity against Renilla luciferase activity.

\section{Western blot analysis}

Western blotting was performed as described previously $^{48,49}$. Cells were lysed using RIPA buffer with Phenylmethanesulfonyl fluoride and phosphatase inhibitors, and total proteins were extracted after transfection. The Protein was separated by $10 \%$ SDS-polyacrylamide gel electrophoresis and then electro-transferred to polyvinylidene difluoride membrane. The membranes were then collected and blocked by the blocking buffer containing $5 \%$ nonfat milk for $1 \mathrm{~h}$. Subsequently, the bands were incubated with primary antibodies overnight at $4{ }^{\circ} \mathrm{C}$ and then with secondary antibodies for $1 \mathrm{~h}$ at room 
temperature. The bands were detected by the Pro-lighting HRP regent and semi-quantitatively analyzed by ImageJ.

\section{In vivo tumor growth assay}

Four- to 5-week-old NOD SCID male mice were purchased from Beijing Vital River Laboratory Animal Technology Co. Ltd (Beijing, China). Eight mice were randomly divided into two groups $(n=4)$. 786-O cells were infected with LV3-hsa-miR-153-5p inhibitor sponge or LV3-NC (GenePharma, Shanghai, China). 786-O cells with two different transfections (each $5 \times 10^{6}$ cell) were suspended in $200 \mu \mathrm{l} \mathrm{PBS}$ and injected subcutaneously into the right armpit of two groups of mice, respectively. Tumor size was monitored every 6 days and the volumes were measured using the following formula: volume $=1 / 2$ length $\times$ width $^{2}$. The mice were killed and tumors were collected 2 months post injection. All animal procedures were approved by the Ethical Committee of Shandong University.

\section{Bioinformatic analysis}

TCGA data of ccRCC, including RNA sequencing and clinical data for 522 tumors and 72 normal tissues, were downloaded from the Genomic Data Commons Data Portal $^{50}$. Differential expression analysis and volcano plots graphing were performed using $\mathrm{R}$ programming language, which is a software environment for graphics and statistical computing. KEGG pathways enrichment analysis and GO enrichment analysis was performed to investigate the miR-153-5p-related signaling. GEPIA ${ }^{51}$, LinkedOmics ${ }^{52}$, and OncoLnc ${ }^{14}$ websites were employed to conduct the expression analysis and survival analysis of miR-153-5p or AGO1.

\section{Statistical analysis}

Statistical differences between two groups were assessed using Student's $t$-test and one-way analysis of variance was employed to identify the statistical differences among multiple groups. For the data not meet normal distribution, the Mann-Whitney test and Kruskal-Wallis test were used, respectively. Pearson's $X^{2}$-test was used to compare clinicopathological features between groups when the data conformed to normal distribution. Kaplan-Meier method was employed to plot survival curves by the log-rank test. Differences with a two-tailed $P<0.05$ were considered significant. GraphPad Prism Software was employed for statistical analysis.

\section{Acknowledgements}

This work was supported by a grant of the National Natural Science Foundation of China (number 81672522), two grants of Cheeloo College of Medicine of Shandong University-Karolinska Institute Cooperative Research Projects (number SDU-KI-2020-8 and number SDU-KI-2020-10). This work was supported by a grant of the National Natural Science Foundation of China (No. 81672522), two grants of Cheeloo College of Medicine of Shandong
University- Karolinska Institute Cooperative Research Projects (No. SDU-KI-20208 and No. SDU-KI-2020-10).

\section{Author details}

'Department of Urology, Qilu Hospital, Cheeloo College of Medicine, Shandong University, Jinan, Shandong 250012, China. ${ }^{2}$ Laboratory of Basic Medical Sciences, Qilu Hospital of Shandong University, Jinan, Shandong 250012, China. ${ }^{3}$ Center for Reproductive Medicine, Cheeloo College of Medicine, Shandong University, Jinan, Shandong 250012, China

\section{Author contributions}

This study was designed by D.Y. and S.Z. Experiments were conducted by Z.L. and S.Z. Data analysis was accomplished by S.Z. and Z.L. The manuscript was written by Z.L. and D.Y., and approved by all the authors.

Conflict of interest

The authors declare that they have no conflict of interest.

Ethics statement

All experimental and animal procedures were approved by the Ethical Committee of Shandong University.

\section{Publisher's note}

Springer Nature remains neutral with regard to jurisdictional claims in published maps and institutional affiliations.

Supplementary Information accompanies this paper at (https://doi.org/ 10.1038/s41419-020-03306-y).

Received: 17 September 2020 Revised: 24 November 2020 Accepted: 25 November 2020

Published online: 04 January 2021

\section{References}

1. Siegel, R. L., Miller, K. D. \& Jemal, A. Cancer statistics, 2018. CA Cancer J. Clin. 68, 7-30 (2018).

2. Siegel, R. L., Miller, K. D. \& Jemal, A. Cancer statistics, 2016. CA Cancer J. Clin. 66 , 7-30 (2016).

3. Minardi, D. et al. Recent aspects of sunitinib therapy in patients with metastatic clear-cell renal cell carcinoma: a systematic review of the literature. Curr. Urol. Rep. 16, 3 (2015)

4. Ljungberg, B. et al. EAU guidelines on renal cell carcinoma: 2014 update. Eur. Urol. 67, 913-924 (2015).

5. Jafri, M. A., Al-Qahtani, M. H. \& Shay, J. W. Role of miRNAs in human cancer metastasis: Implications for therapeutic intervention. Semin. Cancer Biol. 44, 117-131 (2017).

6. Ambros, $\mathrm{V}$. The evolution of our thinking about microRNAs. Nat. Med. 14, 1036-1040 (2008).

7. Rupaimoole, R. \& Slack, F. J. MicroRNA therapeutics: towards a new era for the management of cancer and other diseases. Nat. Rev. Drug Discov. 16, 203-222 (2017).

8. Li, Y. et al. MicroRNA-200b is downregulated and suppresses metastasis by targeting LAMA4 in renal cell carcinoma. EBioMedicine 44, 439-451 (2019).

9. Xiao, W. Wang, X., Wang, T. \& Xing, J. MiR-223-3p promotes cell proliferation and metastasis by downregulating SLC4A4 in clear cell renal cell carcinoma. Aging 11, 615-633 (2019).

10. Chen, Z. Y. et al. MiR-543 promotes cell proliferation and metastasis of rena cell carcinoma by targeting Dickkopf 1 through the Wnt/beta-catenin signaling pathway. J. Cancer 9, 3660-3668 (2018).

11. Shang, J. et al. CircPAN3 mediates drug resistance in acute myeloid leukemia through the miR-153-5p/miR-183-5p-XIAP axis. Exp. Hematol. 70, 42-54.e43 (2019).

12. Zhou, B. et al. CircPCNXL2 sponges miR-153 to promote the proliferation and invasion of renal cancer cells through upregulating ZEB2. Cell Cycle 17, 2644-2654 (2018)

13. Sekino, Y. et al. Uc.416 + A promotes epithelial-to-mesenchymal transition through miR-153 in renal cell carcinoma. BMC Cancer 18, 952 (2018). 
14. Jordan, A. OncoLnc: linking TCGA survival data to mRNAs, miRNAs, and IncRNAs. PeerJ Comput. Sci. 2, e67 https://doi.org/10.7717/peerj-cs.67 (2016).

15. Escudier, B. et al. Renal cell carcinoma: ESMO Clinical Practice Guidelines for diagnosis, treatment and follow-up. Ann. Oncol. 27, v58-v68 (2016).

16. Moch, H., Cubilla, A. L., Humphrey, P. A., Reuter, V. E. \& Ulbright, T. M. The 2016 WHO Classification of Tumours of the Urinary System and Male Genital Organs-Part A: renal, penile, and testicular tumours. Eur. Urol. 70, 93-105 (2016).

17. Li, Z. et al. Prognostic significance of cyclin D1 expression in renal cell carcinoma: a systematic review and meta-analysis. Pathol. Oncol. Res. https://doi. org/10.1007/s12253-019-00776-0 (2019)

18. Lee, H. C. et al. Radiation-inducible miR-770-5p sensitizes tumors to radiation through direct targeting of PDZ-binding kinase. Cell Death Dis. 8, e2693 (2017).

19. Majem, B. et al. MicroRNA-654-5p suppresses ovarian cancer development impacting on MYC, WNT and AKT pathways. Oncogene 38, 6035-6050 (2019).

20. Chen, Y. et al. Silencing of microRNA-708 promotes cell growth and epithelialto-mesenchymal transition by activating the SPHK2/AKT/beta-catenin pathway in glioma. Cell Death Dis. 10, 448 (2019).

21. Joo, L. J. S. et al. RET kinase-regulated microRNA-153-3p improves therapeutic efficacy in medullary thyroid carcinoma. Thyroid 29, 830-844 (2019).

22. Liang, $\mathrm{H}$. et al. miR-153 inhibits the migration and the tube formation of endothelial cells by blocking the paracrine of angiopoietin 1 in breast cancer cells. Angiogenesis 21, 849-860 (2018)

23. Huang, Q. et al. miR-153 suppresses IDO1 expression and enhances CAR T cell immunotherapy. J. Hematol. Oncol. 11, 58 (2018).

24. Chang, A. C., Lien, M. Y., Tsai, M. H., Hua, C. H. \& Tang, C. H. WISP-1 promotes epithelial-mesenchymal transition in oral squamous cell carcinoma cells via the miR-153-3p/Snail axis. Cancers 11, https://doi.org/10.3390/ cancers11121903 (2019).

25. Wang, $\mathrm{H}$. et al. Downregulation of FAP suppresses cell proliferation and metastasis through PTEN/PI3K/AKT and Ras-ERK signaling in oral squamous cell carcinoma. Cell Death Dis. 5, e1155 (2014).

26. Wang, M. et al. SYNJ2BP promotes the degradation of PTEN through the lysosome-pathway and enhances breast tumor metastasis via PI3K AKT/ SNAl1 signaling. Oncotarget 8, 89692-89706 (2017).

27. Li, H. B. et al. Long non-coding RNA-MALAT1 mediates retinal ganglion cell apoptosis through the PI3K/Akt signaling pathway in rats with glaucoma. Cell. Physiol. Biochem. 43, 2117-2132 (2017).

28. Guo, H. et al. The PI3K/AKT pathway and renal cell carcinoma. J. Genet. Genomics 42, 343-353 (2015).

29. Hutvagner, G. \& Simard, M. J. Argonaute proteins: key players in RNA silencing. Nat. Rev. Mol. Cell Biol. 9, 22-32 (2008).

30. Ambros, V. The functions of animal microRNAs. Nature 431, 350-355 (2004).

31. Faehnle, C. R. \& Joshua-Tor, L. Argonautes confront new small RNAs. Curr. Opin. Chem. Biol. 11, 569-577 (2007).

32. Bartel, D. P. MicroRNAs: target recognition and regulatory functions. Cell 136 215-233 (2009).

33. Sasaki, T., Shiohama, A., Minoshima, S. \& Shimizu, N. Identification of eight members of the Argonaute family in the human genome. Genomics $\mathbf{8 2}$ 323-330 (2003).
34. Fabian, M. R., Sonenberg, N. \& Filipowicz, W. Regulation of mRNA translation and stability by microRNAs. Annu. Rev. Biochem. 79, 351-379 (2010).

35. Chen, Z. et al. Hypoxia-responsive miRNAs target argonaute 1 to promote angiogenesis. J. Clin. Investig. 123, 1057-1067 (2013).

36. Voller, D. et al. Argonaute family protein expression in normal tissue and cancer entities. PLOS ONE 11, e0161165 (2016).

37. Li, L., Yu, C., Gao, H. \& Li, Y. Argonaute proteins: potential biomarkers for human colon cancer. BMC Cancer 10, 38 (2010).

38. Rabien, A. et al. Diagnostic and prognostic potential of microRNA maturation regulators Drosha, $\mathrm{AGO} 1$ and $\mathrm{AGO} 2$ in urothelial carcinomas of the bladder. Int. J. Mol. Sci. 19, https://doi.org/10.3390/ijms19061622 (2018).

39. Li, Y. et al. MiR-770 suppresses the chemo-resistance and metastasis of triple negative breast cancer via direct targeting of STMN1. Cell Death Dis. 9, 14 (2018).

40. Krebs, M. et al. miR-221-3p regulates VEGFR2 expression in high-risk prostate cancer and represents an escape mechanism from sunitinib in vitro. J. Clin. Med. 9, https://doi.org/10.3390/jcm9030670 (2020)

41. Chamorro-Jorganes, A., Araldi, E. \& Suárez, Y. MicroRNAs as pharmacological targets in endothelial cell function and dysfunction. Pharmacol. Res. 75, 15-27 (2013).

42. Qiao, J. et al. MicroRNA-153 improves the neurogenesis of neural stem cells and enhances the cognitive ability of aged mice through the notch signaling pathway. Cell Death Differ. 27, 808-825 (2020).

43. Zhang, W. et al. MicroRNA-153 decreases tryptophan catabolism and inhibits angiogenesis in bladder cancer by targeting indoleamine 2,3-dioxygenase 1. Front Oncol. 9, 619 (2019).

44. Santoni, M. et al. Bone metastases in patients with metastatic renal cell carcinoma: are they always associated with poor prognosis? Journal of experimental \&. Clin. Cancer Res. 34, 10 (2015).

45. Santini, D. et al. Natural history of malignant bone disease in renal cancer: final results of an Italian bone metastasis survey. PLoS ONE 8, e83026 (2013).

46. Zhang, N. et al. MicroRNA-30a suppresses breast tumor growth and metastasis by targeting metadherin. Oncogene 33, 3119-3128 (2014).

47. Heckman, K. L. \& Pease, L. R. Gene splicing and mutagenesis by PCR-driven overlap extension. Nat. Protoc. 2, 924-932 (2007).

48. Li, Z., Wang, H., Wang, Q. \& Sun, J. Buyang Huanwu decoction vigorously rescues PC12 cells against 6-OHDA-induced neurotoxicity via Akt/GSK3beta pathway based on serum pharmacology methodology. Rejuvenation Res. 19, 467-477 (2016)

49. Li, Z. et al. Ethanol extract of Patrinia scabiosaefolia induces the death of human renal cell carcinoma $786-0$ cells via SIRT-1 and mTOR signaling mediated metabolic disruptions. Oncol. Rep. 39, 764-772 (2018).

50. Comprehensive molecular characterization of clear cell renal cell carcinoma. Nature 499, 43-49, https://doi.org/10.1038/nature12222 (2013).

51. Tang, Z. et al. GEPIA: a web server for cancer and normal gene expression profiling and interactive analyses. Nucleic Acids Res. 45, W98-W102 (2017).

52. Vasaikar, S. V., Straub, P., Wang, J. \& Zhang, B. LinkedOmics: analyzing multiomics data within and across 32 cancer types. Nucleic Acids Res. 46, D956-D963 (2018). 